\title{
The lack of information system application is the shortcomings of COVID-19 site prevention and control
}

\author{
Author: \\ Cheng Yuan yuan（程园园） \\ Address for correspondence: \\ E-mail:henuhh@outlook.com \\ Affiliation: \\ Henan University Huaihe hospital, Kaifeng City, Henan Province, China.
}

\begin{abstract}
Background: COVID-19 on-site prevention and control refers to the large-scale crowd vaccination and nucleic acid testing activities organized for the prevention and control of COVID-19. Due to factors such as gathering of people and disorganization, there are risk of cross-infection of COVID19 at the event site. Methods: The organizational structure, characteristics, implementation process and system advantages of the COVID-19 on-site prevention and control information system were introduced, and the application basis and problems of the COVID-19 on-site prevention and control information system were discussed. Results: The COVID-19 on-site prevention and control information system is standardized, specialized, intelligent, and easy to replicate, it can solve problems such as the lack of experience in community prevention and control, the problem of prolonged crowd gathering and cross-infection, the problem of low manual writing efficiency, and the psychological problem of individual and group anxiety. Conclusions: The omission of the important element of on-site prevention and control informatization in the prevention and control strategy has led to the lack of application of COVID-19 prevention and control information system, which has become the short board of COVID-19 on-site prevention and control. The informatization should be paid to attention and the use of COVID-19 prevention and control information system in COVID-19 field prevention and control to make up for the shortcomings of epidemic prevention and control.
\end{abstract}

Keywords: COVID-19; informatization; information system; screening

The strategies used in China to deal with COVID-19 are to insist on implementing a strict prevention and control model ${ }^{[1,2]}$, starting from three aspects simultaneously: controlling the source of infection, cutting off the transmission route, and protecting susceptible people, with specific measures including that:

1. Suspension of public transportation, such as flight meltdown. 
2. Cessation or restriction of business premises, such as cessation or restriction of restaurants, cinemas, and tourist attractions.

3. Health codes and communication big data trip card (trip code) management.

4. Big data with epidemiological survey to identify close contacts and sub-close contacts of cases in the population.

5. Centralized isolation and home isolation at isolation sites.

6. Community closure management and urban closure and control management.

7. Differential management of low, medium and high risk areas.

8. Mass population screening activities for COVID-19 or nucleic acid testing, etc.

Among them, the large-scale crowd vaccination and nucleic acid testing screening activities organized for the prevention and control of COVID-19, i.e. COVID-19 on-site prevention and control, is one of the few permitted crowd gathering activities at present. There is a risk of COVID19 cross-infection at the event site due to factors such as crowd gathering and disorganization.

In July 2021, there were successive incidents in Nanjing City and Yangzhou City ${ }^{[4-12]}$, China, in which participants of nucleic acid testing were infected with SARS-CoV-2 at the site of communityorganized nucleic acid testing, especially at the nucleic acid testing site in Union Village, Wantou Town, Yangzhou City, which was not set up in a standardized manner and the site was disorganized, leading to the fact that 38 people were infected in the non-infectious person who visited the site on July 29. As of 24:00 on August 9, the 38 people were diagnosed with COVID-19 cases. From July 28 to August 9 at 24:00, Yangzhou City reported a total of 394 confirmed local cases, of which the number of cases that caused illness after infection was acquired at the prevention and control site on July 29 accounted for $9.6 \%{ }^{\left[4^{-} 12\right]}$.

The on-site prevention and control of COVID-19 is generally coordinated by the COVID-19 epidemic prevention and control command department, with vaccination and nucleic acid screening technical services provided by medical and health institutions and implementation organized by the grassroots community sector. Limited by the level of expertise of community staff and experience in on-site organization, the loopholes likely to occur at the COVID-19 on-site prevention and control. The occurrence of on-site cross-infection incidents of nucleic acid testing, on the one hand, has the problems of irregular setting of nucleic acid testing sites and chaotic on-site organization in the grassroots community sector, and on the other hand, there are also problems of omissions in the COVID-19 on-site prevention and control strategyies, which expose the problems of weak awareness of informatization prevention and control and insufficient informatization expertise in the COVID-19 epidemic prevention and control command department. The omission of the important element of on-site prevention and control informatization from the prevention and control 
strategies have led to the lack of application of COVID-19 prevention and control information system, which has become the short board of COVID-19 on-site prevention and control.

The key to COVID-19 on-site prevention and control informatization lies in the application of COVID-19 on-site prevention and control information system(PCIS), the organizational structure of PCIS includes.

1. Data processing center: It is used to collect, process and store information of those who receive COVID-19 vaccination and nucleic acid test, and provide proof of COVID-19 vaccination and nucleic acid tests. It is generally maintained by the local big data management service center.

2. Information collection system: It includes two parts: "registration" subsystem and "sub-inspection disposal" subsystem. The "registration" subsystem undertakes the function of collecting the information of the reservation holder and transmits the information to the information display system. In addition to checking the information of reservation holders, the "sub-inspection disposal" sub-system can also collect the data of the prevention and control site from time to time, intelligently divert the reservation holders to different disposal windows according to the labor intensity and efficiency of the staff, and remind the site staff to change shifts, take a break, and do the disinfection work on site, etc. The information collection system aggregates the information collected at the site and transmits it to the data processing center through the Internet.

3. Information display system: also called on-site calling system, according to the progress of onsite activities, it use voice call, large screen synchronous display and other ways to queue and call numbers by serial number, or issue other reminder information.

\section{The implementation process of the PCIS application.}

1. Individuals participating in vaccination or nucleic acid testing should install and use the local unified APP (or applet) on their cell phones, fill in the necessary information, such as name, identification information (e.g., ID number, passport number, etc.), contraindications, and cell phone number, and send the completed information to the data processing center for appointment registration. This step can also be done at the prevention and control site, with the assistance of staff using the information collection system.

2. After receiving the appointment registration information filled out by the individual through the APP, the data processing center sends back to the reservation holder a QR code that records the relevant information of the reservation holder. The information contained in this QR code includes the information declared by the person making the appointment and other information, such as whether the person has received COVID-19 vaccine, the type and batch of COVID-19 vaccine received, etc. This information can be read out by the information collection system without being connected to the Internet.

3. When the person making the appointment arrives at the COVID-19 prevention and control site (set-up point) and presents this QR code, the "registration" subsystem of the information collection 
system reads the QR code and automatically generates a serial number with a barcode, or prints it out and gives it to the person, or the person keeps the serial number and barcode by taking pictures, and the serial number is entered into the information display system at the same time. The serial number will be entered into the information display system at the same time. When the number of registered persons reaches the limit of the set-up point, the sub-system of "registration" will stop the business processing of this set-up point.

4. The information display system reminds the reservation holders of the matters they need to pay attention to by means of voice calling and large screen display, how long the reservation holders of the corresponding serial number need to wait, which sub-inspection window to carry out vaccination or nucleic acid testing, the remaining places that can accept registration, etc. This information can also be pushed to the reservation holders' cell phones from time to time by paying attention to the instant messaging APP, live platform APP, etc.

5. According to the prompts of the information display system, the reservation holder will hold the barcode of the serial number generated by the "registration" subsystem and arrive at the corresponding sub-inspection and disposal window. Then the staff will use the "sub-inspection disposal" subsystem of the COVID-19 on-site information collection system to scan the serial number barcode, and read the barcode information of COVID-19 vaccine specification, batch barcode or accounting test tube number, bind both information for traceability, and check the information of the reservation holder again before conducting sub-testing and disposal. Ensure that the vaccination or accounting test work is done with: the correct reservation holder, the correct registration registrant, the correct vaccine type, the correct vaccine dose, and the correct accounting collection.

\section{The implementation process of the PCIS is shown in Figure 1.}




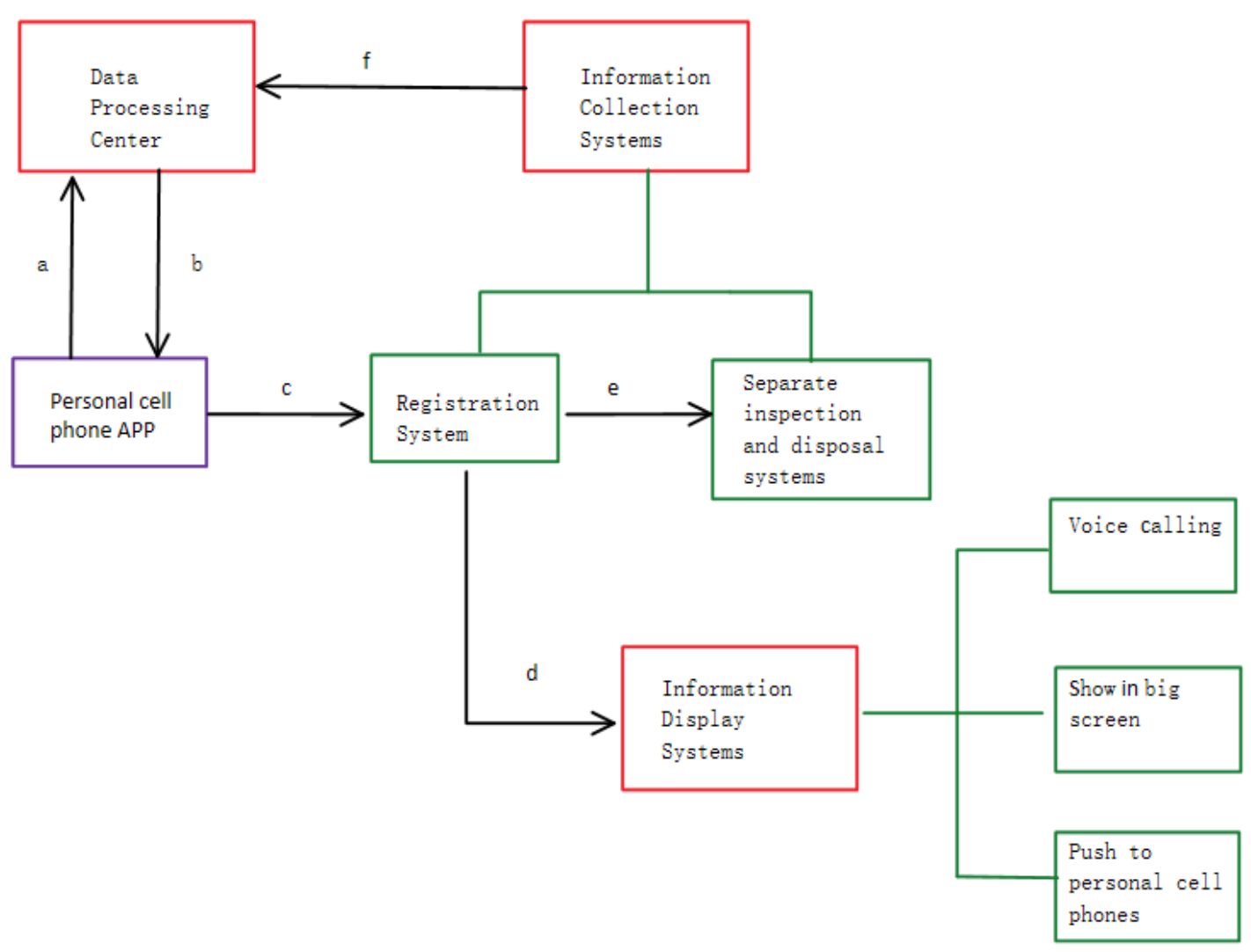

Figure 1: The implementation process of the PCIS.

a: Install and use the local unified APP (or applet) on your cell phone, fill in your personal information such as name, identity information (e.g., ID number, passport number), contraindications, cell phone number, etc., and send the filled-in information to the data processing center for appointment registration.

$\mathrm{b}$ : The data processing center sends back a $\mathrm{QR}$ code with the relevant information of the reservation holder.

c: When the reservation holder arrives at the site and presents the QR code, the QR code will be read by the "registration" sub-system of the information collection system and a serial number with barcode will be automatically generated.

d: This serial number at the same time into the information display system.

e: The sub-system of "sub-inspection disposal" scans and reads the barcode of the serial number, and reads the barcode of the specification and batch of COVID-19 vaccine or the barcode information of the accounting test tube number, so that the two information can be bound to each other for traceability, and then sub-tests and disposes of the information after checking the appointment again.

f: The information collected on site are summarized and transmitted to the data processing center.

\section{Features of PCIS.}

1. Specialization: The design of PCIS integrates professional theoretical knowledge and practical application results, and its process settings reflect the professionalism of COVID-19 on-site prevention and control. 
2. Standardization: The PCIS uses standardized processes and evaluation systems, and can achieve consistent results when used at different times, in different areas, and among different groups of people.

3. Intelligent: The PCIS incorporates scientific algorithms, which can calculate the collected on-site data, make timely feedback or issue instructions, intelligently protect the rights and interests of staff, divert the work pressure of different positions, divert the order of the site, and constantly adjust and balance between work intensity and work efficiency.

4. Easy to replicate: The PCIS is easy to replicate and easy to promote and use in different areas or communities, and at the same time, its specialization, standardization and intelligence will be quickly promoted and applied because of easy replication. It is important to note that design flaws, errors and vulnerabilities in the PCIS can be replicated quickly and synchronized, so a mechanism for feedback and correction of design flaws, errors and vulnerabilities needs to be added to the information system, which requires a deep understanding.

\section{The PCIS can solve or mitigate the following problems.}

1. The problem of insufficient experience in community prevention and control: The work of COVID-19 on-site prevention and control generally initiated and organized by the COVID-19 epidemic prevention and control command department, it is ultimately implemented by the grassroots community departments, which often lack experience in organizing large-scale crowd prevention and control and have insufficient reserves in the knowledge base and informatization cognition of infectious disease prevention and control, and there are prone to errors. The PCIS, while the system is established, it incorporates standardized processes,specialized norms, and intelligent algorithms. When implemented according to the steps prompted by the system, the standardization, specialization, and intelligence are then implemented, thus solving the problems of irregular settings and confusing on-site organization caused by the lack of experience in on-site prevention and control in community departments.

2. The problems of a large number of crowed gathering for a long time and cross-infection: After the reservation holder has collected the QR code information at the site and got the serial number, he can scatter or even leave the site temporarily, and only needs to pay attention to the information prompt of the site information display system or cell phone APP and appear in front of the disposal window for vaccination or nucleic acid collection on time, which greatly reduces the risk of crossinfection caused by a large number of crowed gathering at the site for a long time.

3. The problem of low efficiency of manual writing and long detention time at the site: The information collection system uses a scanner to scan bar codes (including two-dimensional codes) to collect information 60 to 100 times more efficiently than filling out forms manually, which can solve the problem of high recognition error rate caused by factors such as scribbles and irregular writing, and can solve the problem of low efficiency of manual writing at the site; paperless and contactless operation can further reduce the risk of cross-infection caused by high-density gathering and long detention time of personnel at the site.

4. Psychological problems of individual and group anxiety : When knowing the serial number and 
progress, the reservation holders can predict and reasonably arrange their waiting time, and the information are publicized through the large screen reflects that the principles of openness, fairness and acceptance of supervision, which is a necessary rather than an optional link, it can alleviate the psychological problems of individual and group anxiety ${ }^{[13-19]}$.

\section{Application basis of PCIS.}

In recent years, China is vigorously implementing the "Internet+" initiative, i.e., "Internet + various traditional industries", to create new advantages in the digital economy ${ }^{[20]}$. In the medical field, the "Internet + medical" initiative is being implemented, i.e., the development of medical informatization and the creation of digital hospitals. Hospitals at all levels have built hospital information systems(HIS) according to unified standards and achieved informatization standards appropriate to the level of the hospital.

For example, in the outpatient halls of hospitals above the second level, self-help equipments are generally required to realize the functions of patient self-registration, payment, querying medical information, printing examination and test results, etc.; queuing and calling equipments are set up to process and display information about patients waiting in line for consultation, examination and medication, so that patients can understand and arrange their own waiting time; information collection systems are used to read patients' consultation card information and generate outpatient cases, etc. In order to avoid repetitive writing of items and improve the efficiency of information input. The application of medical informatization has achieved positive results in optimizing the flow of medical treatment, improving the efficiency of outpatient services, and enhancing patient satisfaction. The application results and experience of "Internet+" are a solid foundation for the application of PCIS. It is only necessary to add modules suitable for COVID-19 on-site prevention and control in the HIS to play an important role of information system in COVID-19 on-site prevention and control.

\section{Main problems in the application of PCIS.}

Health care institutions have a practical basis for the application of information systems, which can be beneficial for on-site prevention and control of COVID-19 in large populations if they provide prevention and control technologies in conjunction with the use of information system applications for prevention and control sites. However, the information system is not practically applied even in the prevention and control sites that are organized and implemented by health care institutions. The main reason is that the organizers of health care institutions mechanically understand and implement HIS, only use the modules integrated into the HIS, but do not know how to actively add and modify the prevention and control modules that are not in the HIS, especially the prevention and control information collection subsystem and the prevention and control information display subsystem, as well as the prevention and control processes that reflect standardization, specialization, and intelligence. The COVID-19 epidemic prevention and control command department also have the problem of insufficient awareness of informatization prevention and control, and the problem of insufficient awareness of PCIS, so that the application of information systems are missing in on-site crowd prevention and control, resulting in the short board effect of on-site crowd prevention and 
control.

\section{Oil barrel principle.}

There are an "oil barrel principle" in COVID-19 prevention and control site: if we call the capabilities of management of COVID-19 prevention and control site a "barrel full of gasoline", and the source of COVID-19 infection a "spark", then the irregular setup and disorganized management of the site is the overturned oil barrel. When COVID-19 prevention and control site have both spilled oil bucket and "spark", it is like gasoline meets fire and burns rapidly, and the problem of cross-infection is difficult to control. When the COVID-19 prevention and control site have only spilled oil from the barrels or only "spark", that is, there is "oil" but no "spark" or "spark" without "oil", and will not cause cross-infection of COVID-19. If the COVID-19 prevention and control site have irregular settings and disorganized management, but there are no source of COVID-19 infection, it may be largely due to the presence of "oil" without "fire". We should not hope that there are "oil" without "fire", and we should be prepared to have "fire" and "oil". The "oil barrel principle" reminds us that we should pay attention to the management of the "barrel full of gasoline", strengthen the awareness of COVID-19 informatization prevention and control, establish and use standardized, professional and intelligent PCIS, or on the basis of the existing HIS, the COVID-19 prevention and control module should added through expansion and transformation, and immediately put into use to make up for the shortcomings of COVID-19 on-site prevention and control, which are an important prevention and control strategies that should not be ignored. Taking COVID-19 prevention and control as an opportunity to study and develop construction standards for crowed prevention and control information systems for infectious diseases and epidemics are an important prevention and control strategies that should not be neglected.

\section{References.}

[1] Summary of policy measures for epidemic prevention and control across China [EB/OL]. http://www.gov.cn/zhuanti/2021yqfkgdzc/index.htm.[2021-10-9]

[2] COVID-19 Prevention and Control Program (8th Edition) [EB/OL]. https://oss.henan.gov.cn/typtfile/20210825/01bf2af26d1543a694203718d06e197f.pdf.[2 021-08-11]

[3] New confirmed cases of COVID-19 in Nanjing from 0:00 to 24:00 on July 31 [EB/OL]. http://wjw.nanjing.gov.cn/njswshjhsywyh/202108/t20210801_3089858.html.2021-08$\underline{01]}$

[4] Epidemic notification: 26 new confirmed cases of COVID-19 in Yangzhou [EB/OL].http://wjw.yangzhou.gov.cn/yzwshjh/ywkd/202108/9a16adef18e3488db64a3e b41314e262.shtml. [2021-08-02].

[5] Epidemic notification: 40 new confirmed cases of COVID-19 in Yangzhou City $[\mathrm{EB} / \mathrm{OL}]$. 
http://wjw.yangzhou.gov.cn/yzwshjh/ywkd/202108/fa41d8efb3644fd68393c0aed478f03 e.shtml.[2021-08-03].

[6] Epidemic notification: 32 new confirmed cases of COVID-19 in Yangzhou City [EB/OL].

http://wjw.yangzhou.gov.cn/yzwshjh/ywkd/202108/d62c0e11801f4012b58774ff64b89b ac.shtml.[2021-08-04]

[7] Epidemic notification: 36 new confirmed cases of COVID-19 in Yangzhou City [EB/OL].

http://wjw.yangzhou.gov.cn/yzwshjh/ywkd/202108/f624c59b2d484313bad13aa5a4bd9b e0.shtml.[2021-08-05]

[8] Epidemic notification: 58 new confirmed cases of COVID-19 in Yangzhou City [EB/OL].

http://wjw.yangzhou.gov.cn/yzwshjh/ywkd/202108/073cd0cd0856473a943636e9a33e8 Obe.shtml.[2021-08-06]

[9] Epidemic notification: 52 new confirmed cases of COVID-19 in Yangzhou City. [EB/OL].http://wjw.yangzhou.gov.cn/yzwshjh/ywkd/202108/b539c3eb401a4e148bd58 8b7afc12475.shtml. [2021-08-07]

[10] Epidemic notification: 36 new confirmed cases of COVID-19 in Yangzhou City [EB/OL].

http://wjw.yangzhou.gov.cn/yzwshjh/ywkd/202108/c60718f241a0474986e20bcac66def 7b.shtml.[2021-08-08 ]

[11] Epidemic notification: 38 new confirmed cases of COVID-19 in Yangzhou [EB/OL]. http://wjw.yangzhou.gov.cn/yzwshjh/ywkd/202108/ff6674b99a264d37814af7d34b84b5 96.shtml.[2021-08-09]

[12] Epidemic notification: 48 new confirmed cases of COVID-19 in Yangzhou City [EB/OL].http://wjw.yangzhou.gov.cn/yzwshjh/ywkd/202108/a9fa72edc27e435abad92c e9a1d77955.shtml.[2021-08-10 ]

[13] Huang Y, Zhao N. Generalized anxiety disorder, depressive symptoms and sleep quality during COVID-19 outbreak in China: a web-based cross-sectional survey[J]. Psychiatry research, 2020, 288: 112954.

[14] Cao W, Fang Z, Hou G, et al. The psychological impact of the COVID-19 epidemic on college students in China[J]. Psychiatry research, 2020, 287: 112934.

[15] Gao J, Zheng P, Jia Y, et al. Mental health problems and social media exposure during COVID-19 outbreak[J]. Plos one, 2020, 15(4): e0231924.

[16] Wang X, Hegde S, Son C, et al. Investigating mental health of US college students during the COVID-19 pandemic: cross-sectional survey study[J]. Journal of medical Internet research, 2020, 22(9): e22817. 
[17] Kwong A S F, Pearson R M, Adams M J, et al. Mental health before and during the COVID-19 pandemic in two longitudinal UK population cohorts[J]. The British Journal of Psychiatry, 2021, 218(6): 334-343.

[18] Husky M M, Kovess-Masfety V, Swendsen J D. Stress and anxiety among university students in France during Covid-19 mandatory confinement[J]. Comprehensive Psychiatry, 2020, 102: 152191.

[19] Bäuerle A, Teufel M, Musche V, et al. Increased generalized anxiety, depression and distress during the COVID-19 pandemic: a cross-sectional study in Germany[J]. Journal of Public Health, 2020, 42(4): 672-678.

[20] The State Council on actively promoting the "Internet + " action guidance [EB/OL]. http://www.gov.cn/gongbao/content/2015/content_2897187.htm.[2015-07-04] 


\section{Supplementaries}

\section{Competing Interest Statement}

The authors declare no competing interest.

\section{Clinical Trial}

NCT12345678

\section{Funding Statement}

There is no external funding, and there is no conflict of interests.

\section{Authors Declaration}

All relevant ethical guidelines have been followed; any necessary IRB and/or ethics committee approvals have been obtained and details of the IRB/oversight body are included in the manuscript.

Yes

All necessary patient/participant consent has been obtained and the appropriate institutional forms have been archived.

Yes

I understand that all clinical trials and any other prospective interventional studies must be registered with an ICMJE-approved registry, such as ClinicalTrials.gov. I confirm that any such study reported in the manuscript has been registered and the trial registration ID is provided (note: if posting a prospective study registered retrospectively, please provide a statement in the trial ID field explaining why the study was not registered in advance). 
I have followed all appropriate research reporting guidelines and uploaded the relevant EQUATOR Network research reporting checklist(s) and other pertinent material as supplementary files, if applicable.

Yes

Data Availability Statement:

All data, models, and code generated or used during the study appear in the submitted article. 